\title{
BDNF regulates the expression and secretion of VEGF from osteoblasts via the TrkB/ERK1/2 signaling pathway during fracture healing
}

\author{
ZITAO ZHANG, YAN ZHANG, ZHENGNAN ZHOU, HONGFEI SHI, XUSHENG QIU, JIN XIONG and YIXIN CHEN \\ Department of Orthopedics, The Affiliated Drum Tower Hospital of Nanjing University Medical School, \\ Nanjing, Jiangsu 210008, P.R. China
}

Received August 19, 2015; Accepted August 9, 2016

DOI: $10.3892 / \mathrm{mmr} .2017 .6110$

\begin{abstract}
Brain-derived neurotrophic factor (BDNF), a member of the neurotropic family, is expressed in osteoblast-like cells of a fracture callus, however, its role in fracture healing remains to be fully elucidated. Osteoblasts isolated from Sprague Dawley rats were stimulated by BDNF in a dose- and time-dependent manner. Immunoblotting and immunofluorescence was used to detect the expression and distribution of targeted proteins. The concentration of vascular endothelial growth factor (VEGF) released in medium was determined using an ELISA. PD98059 and K252a were used to investigate the signaling pathways that may be involved. The present study demonstrated that BDNF was involved in fracture repair by controlling the expression and secretion of VEGF from osteoblasts, which predominantly drives angiogenesis during fracture healing. Tropomyosin-related kinase B (TrkB), the specific receptor of BDNF, was shown to be expressed at high levels in the osteoblasts. Following BDNF stimulation, TrkB and extracellular signal-regulated kinase 1/2 (ERK1/2) were rapidly activated. The inhibition of TrkB by K252a decreased the expression and secretion of VEGF, and suppressed the phosphorylation level of ERK1/2. PD98059, an antagonist of ERK1/2, elicited the same effects on VEGF from the BDNF-stimulated osteoblasts, however, it did not affect the phosphorylation of TrkB. In conclusion, during fracture healing, BDNF was found to stimulate the expression and secretion of VEGF from osteoblasts via the TrkB/ERK1/2 signaling pathway.
\end{abstract}

Correspondence to: Dr Yixin Chen, Department of Orthopedics, The Affiliated Drum Tower Hospital of Nanjing University Medical School, 321 Zhongshan Road, Nanjing, Jiangsu 210008, P.R. China E-mail: chenyixin93@126.com

Key words: brain-derived neurotrophic factor, vascular endothelial growth factor, osteoblast, fracture healing

\section{Introduction}

Angiogenesis is an essential process in fracture healing, and defective blood supply at the fracture site is a primary consideration in cases of non-union. Several factors are involved in angiogenesis during the osseous repair cascade in a temporospatial manner, however, vascular endothelial growth factor (VEGF) is of primary importance (1). During facture healing, VEGF is released or secreted from different cells, of which osteoblasts have been extensively investigated although the mechanism is complicated. Zhu et al (2) revealed that activating transcription factor 4 is a novel regulator of the VEGF secretion axis in osteoblasts, whereas Rui et al reported that the intracellular scaffold $\mathrm{G}$ protein-coupled receptor kinase interacting factor- 1 also mediates the release of VEGF from osteoblasts (3). However, previous studies have investigated the role of osteoblasts in the expression and secretion of VEGF (4-6), the mechanism remains to be fully elucidated.

Brain-derived neurotrophic factor (BDNF) is a small basic protein, which is a member of the neurotrophin family of growth factors. BDNF is important in neural development and functions through the activation of its specific receptor, tropomyosin-related kinase B (TrkB) $(7,8)$. In addition, several reports have demonstrated the critical effects of BDNF and TrkB in non-neural cells or tissues, including endothelial cells (ECs), chondrosarcoma cells, intervertebral disc degeneration and cardiac contraction (9-12). With respect to osteoblasts, the function of BDNF/TrkB signaling is diverse. For example, Pinski et al (13) found that TrkB is associated with cell apoptosis, whereas another study characterized the rs6265 polymorphism in BDNF, which regulates its differentiation and osteoblastic activity (14). In addition, BDNF/TrkB is involved in fracture healing. A previous study demonstrated that BDNF is localized at high levels in osteoblast-like cells through use of a mouse fracture healing model (15). In human fracture healing, another study indicated that BDNF and TrkB are expressed in osteoblast-like cells, and are involved in vessel formation and the osteogenic process (16). Although rodent and human histological examinations have revealed that BDNF/TrkB is involved during fracture healing, the underlying mechanism remains to be elucidated. 
Several reports have shown that BDNF can promote VEGF-mediated angiogenesis. Silencing of the BDNF gene in multiple myeloma inhibits osteolytic bone destruction, and reduces angiogenesis and tumor burden (17). ECs expressing high levels of BDNF can facilitate tumor angiogenesis and growth (18). However, in different cells, the downstream signaling molecules of the BDNF axis involved in regulating the secretion of VEGF vary considerably. In chondrosarcoma cells, BDNF increases the expression of VEGF and angiogenesis through the TrkB/phospholipase C (PLC) signaling pathway, whereas in human umbilical vein ECs, BDNF promotes angiogenic tube formation through the generation of oxidative stress $(9,11)$. At present, the role of BDNF in the expression of VEGF in osteoblasts remains to be fully elucidated. In the present study, it was demonstrated that BDNF promoted the expression of VEGF in osteoblasts via activation of the TrkB/extracellular signal-regulated kinase (ERK)1/2 signaling pathway. These results indicated that BDNF is important in fracture healing via VEGF-mediated angiogenesis; thus, it may be a novel therapeutic target for the treatment of fracture non-union.

\section{Materials and methods}

Reagents. Anti-rat phosphorylated (p)-ERK1/2, ERK1/2 and anti-phosphotyrosine 4G10 antibodies were purchased from Cell Signaling Technology, Inc. (Danvers, MA, USA). Anti-rat TrkB antibody, PD98059 and K252a were purchased from Abcam (Cambridge, UK). All secondary antibodies used in the present study were purchased from Beyotime Institute of Biotechnology (Shanghai, China). BDNF was purchased from Peprotech, Inc. (Rocky Hill, NJ, USA).

Isolation, culture and stimulation of rat osteoblasts. A total of 16 Sprague Dawley (SD) rats (age, 1-2 days; male/female, 1:1) were obtained from the Department of Animal Science, Nanjing Medical University (Nanjing, China). Neonatal rats were sacrificed by being submerged in $75 \%$ ethanol for $20 \mathrm{~min}$. The neonatal rats were housing with the temperature $18-26^{\circ} \mathrm{C}$ and the humidity was $40-70 \%$ in dark and quiet conditions and fed by their maternal rats. The present study was approved by the Ethics Committee of The Affiliated Drum Tower Hospital of Nanjing University Medical School (Nanjing, China). Primary neonatal calvarial osteoblasts were isolated from the rats according to a previously described method with minor modification (3). Briefly, under aseptic conditions, the calvaria of the 1-2-day-old SD rats were dissociated and digested in $0.05 \%$ trypsin and $0.1 \%$ collagenase (Thermo Fisher Scientific, Inc., Waltham, MA, USA) for $10 \mathrm{~min}$ at $37.8^{\circ} \mathrm{C}$ in an incubator with $5 \% \mathrm{CO}_{2}$. Following termination of digestion, the cell suspensions were passed through a $70 \mu \mathrm{m}$ mesh Falcon nylon filter. The filtered medium was centrifuged at $250 \mathrm{x} g$ for $10 \mathrm{~min}$ at $20^{\circ} \mathrm{C}$ and resuspended in Dulbecco's modified Eagle's Medium (DMEM; Thermo Fisher Scientific, Inc.). The cells were grown in DMEM supplemented with $10 \%$ fetal bovine serum (FBS; Thermo Fisher Scientific, Inc.), $100 \mathrm{IU} / \mathrm{ml}$ penicillin and $100 \mathrm{IU} / \mathrm{ml}$ streptomycin, and incubated at $37^{\circ} \mathrm{C}$ in a $5 \%$ $\mathrm{CO}_{2}$ atmosphere. Confluent cells at a density of $5.0 \times 10^{4} / \mathrm{ml}$ were passaged in medium with $0.25 \%$ trypsin and EDTA every 3 days and allowed to grow to confluence. At the third generation, BDNF was added to the medium only following serum starvation for $30 \mathrm{~min}$ at concentrations of $0,5,25,50$, 100 and $200 \mathrm{ng} / \mathrm{ml}$ and durations of $0,3,6,2,24$ and $48 \mathrm{~h}$ for VEGF detection and 0, 0.5, 1, 2, 5 and $10 \mathrm{~min}$ for kinase assay. K252a (200 nM) or PD98059 $(25 \mu \mathrm{M})$ was added to the medium $30 \mathrm{~min}$ prior to the stimulation with BDNF. An equal quantity of dimethyl sulfoxide was used for incubation of the cells as a solvent control.

Immunoblotting and immunoprecipitation. The cells were lysed and proteins were extracted using RIPA buffer. The protein concentration was quantified using the Bradford method (19). Proteins (10 $\mu \mathrm{g})$ were separated by $10 \%$ SDS-polyacrylamide gel, and transferred onto polyvinylidene fluoride membranes (EMD Millipore, Billerica, MA, USA). The membranes were incubated overnight at $4^{\circ} \mathrm{C}$ with the following primary antibodies: ERK1/2 (cat. no. 4695; 1:1,000), p-ERK1/2 (cat. no. 4377; 1:1,000), 4G10 (cat. no. 9411; 1:500); TrkB (cat. no. ab5372; 1:2,000). This was followed by the addition of a horseradish peroxidase-linked secondary antibodies (cat. nos. A0208, A0216; 1:1,000) at $37^{\circ} \mathrm{C}$ for $2 \mathrm{~h}$, and electrochemiluminescence visualization of the bands (Beyotime Institute of Biotechnology). Quantification of the bands was performed using Quantity One densitometric analysis software (Bio-Rad Laboratories, Inc., Hercules, CA, USA). For immunoprecipitation, the cell lysate was centrifuged at $10,000 \times \mathrm{g}$ for $10 \mathrm{~min}$ at $4^{\circ} \mathrm{C}$. A proportion of the lysate $(0.5 \mathrm{mg})$ was then incubated with $2 \mu \mathrm{g}$ primary antibody overnight at $4^{\circ} \mathrm{C}$ on a rocker platform. Protein A/G-agarose (Santa Cruz Biotechnology Inc., Dallas, TX, USA) was added and mixed for $2 \mathrm{~h}$ at $4^{\circ} \mathrm{C}$. The precipitate was isolated following centrifugation of the mixture at $1,000 \mathrm{x} \mathrm{g}$ for $30 \mathrm{sec}$ at $4^{\circ} \mathrm{C}$ and discarding the supernatant. The following steps performed were similar to those described above for western blot analysis.

Immunofluoresence. Coverslips were washed with phosphate-buffered saline (PBS) three times and then fixed in $-20^{\circ} \mathrm{C}$ cold methanol for $20 \mathrm{~min}$. Following washing with PBS three times, the cells at a density of $1.0 \times 10^{4} / \mathrm{ml}$ were permeated with $0.3 \%$ Triton $\mathrm{X}-100$ for $10 \mathrm{~min}$, followed by blocking with $\mathrm{PBS}$ at $37^{\circ} \mathrm{C}$ containing $10 \% \mathrm{FBS}$ for $1 \mathrm{~h}$. The coverslips were incubated at $4^{\circ} \mathrm{C}$ overnight with TrkB antibody, which was followed by incubation in fluorescein isothiocyanate $(1: 100)$ at $37^{\circ} \mathrm{C}$ for $1 \mathrm{~h}$. The cells were visualized using a confocal microscope.

Detection of VEGF content. The osteoblasts were cultured in 24-well plates. To investigate the downstream signaling pathways, BDNF treatment was performed; cells at a density of $5.0 \times 10^{5} / \mathrm{ml}$ were pretreated with inhibitors (K252a, $200 \mathrm{nM}$ at $37^{\circ} \mathrm{C}$; PD98059, $25 \mu \mathrm{M}$ at $37^{\circ} \mathrm{C}$ ) for $30 \mathrm{~min}$ prior to the addition of $50 \mathrm{ng} / \mathrm{ml}$ BDNF for $12 \mathrm{~h}$. The medium was extracted and examined immediately. A VEGF (colorimetric ELISA) assay kit (ExCell Biological Products Co., Ltd., Shanghai, China) was used to determine the VEGF content in the culture medium according to the manufacturer's protocol.

Statistical analysis. The data are shown as the mean \pm standard error of the mean. All experiments were repeated three 
A

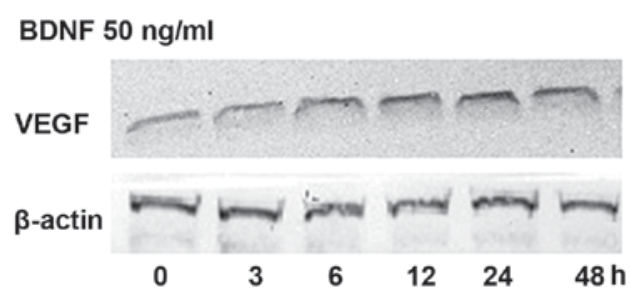

C
B

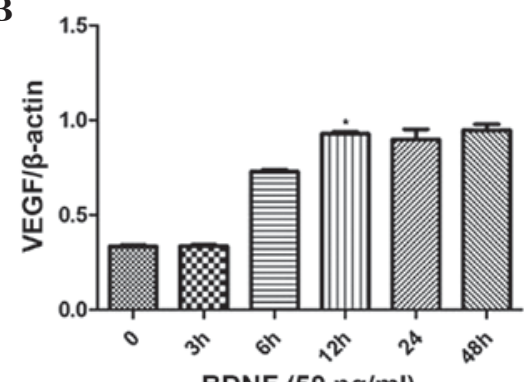

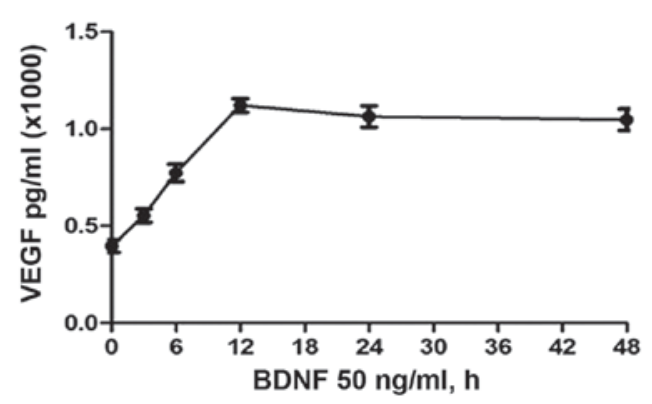

Figure 1. Time-dependent effects of BDNF on the expression and release of VEGF from osteoblasts stimulated by BDNF. Osteoblasts were stimulated by BDNF at a concentration of $50 \mathrm{ng} / \mathrm{ml}$ for $0,3,6,12,24$ and $48 \mathrm{~h}$, respectively. (A) Expression was examined using immunoblotting. (B) Statistical analysis of the immunoblotting results. (C) Concentrations of VEGF in the media were detected using ELISA. Data are presented as the mean \pm standard error of the mean. ${ }^{*} \mathrm{P}<0.05$, compared with 0,3 and $6 \mathrm{~h}$. BDNF treatment. BDNF, brain-derived neurotrophic factor; VEGF, vascular endothelial growth factor.

A

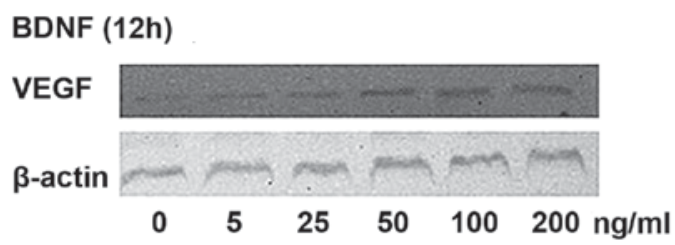

B

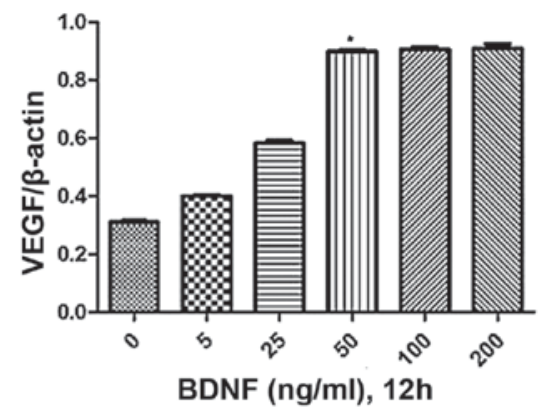

C

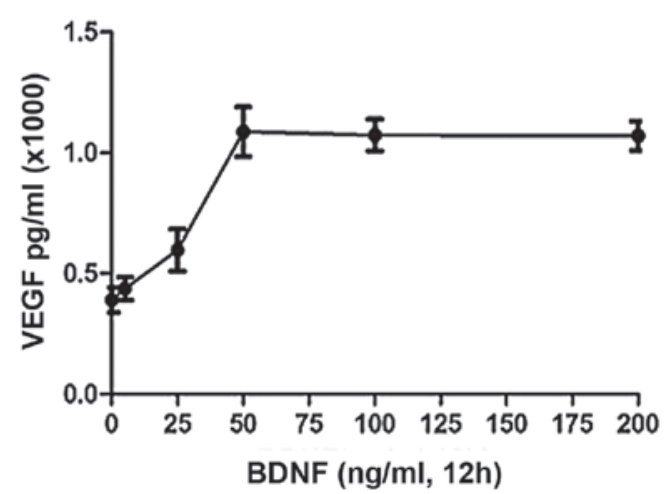

Figure 2. Dose-dependent effects of BDNF on the expression and release of VEGF from osteoblasts stimulated by BDNF. Cells were stimulated for $12 \mathrm{~h}$ with BDNF at concentrations of 0, 25, 50, 100 and $200 \mathrm{ng} / \mathrm{ml}$, respectively. (A) Expression was examined using immunoblotting. (B) Statistical analysis of the immunoblotting results. (C) Concentrations of VEGF in the media were detected using ELISA. Data are presented as the mean \pm standard error of the mean. ${ }^{*} \mathrm{P}<0.05$, compared with 0, 5 and $25 \mathrm{ng} / \mathrm{ml}$. BNDF. BDNF, brain-derived neurotrophic factor; VEGF, vascular endothelial growth factor.

times independently. Student's t-test or one-way analysis of variance were used to compare the differences among the groups using SPSS version 16.0 (SPSS, Inc., Chicago, IL, USA). $\mathrm{P}<0.05$ was considered to indicate a statistically significant difference.

\section{Results}

$B D N F$ promotes the expression and release of VEGF from osteoblasts. ELISA and immunoblotting were utilized to detect the expression and secretion of VEGF. To evaluate the 
A

BDNF 50ng/ml

p-ERK1/2

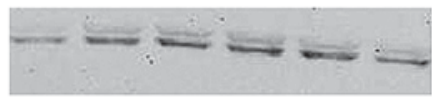

ERK1/2

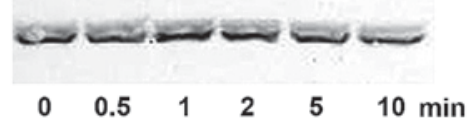

C

IP-TrkB

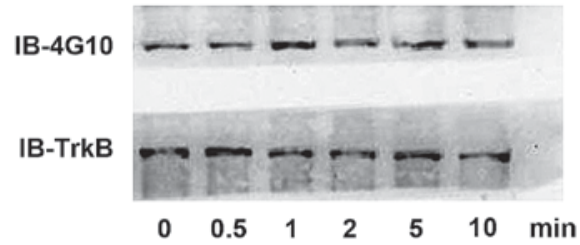

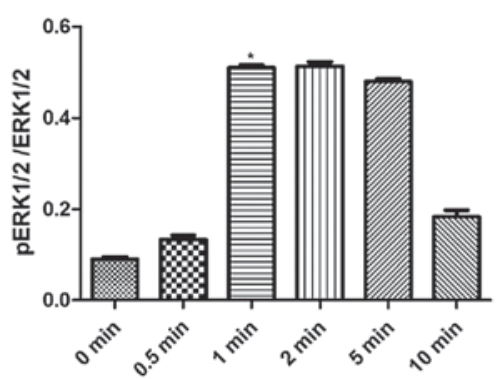

D

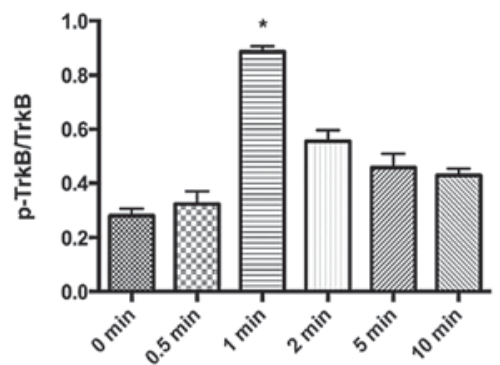

Figure 3. Effects of BDNF on the phosphorylation of TrkB and ERK1/2. (A) Effects of BDNF stimulation on the phosphorylation of ERK1/2 at 0,0.5, 1,2,5 and 10 min with (B) results of statistical analysis. (C) Effects of BDNF stimulation on the phosphorylation of TrkB at 0, 0.5, 1, 2, 5 and 10 min with (D) results of statistical analysis. Data are presented as the mean \pm standard error of the mean. $\mathrm{P}<0.05$, compared with the control group. BDNF, brain-derived neurotrophic factor; TrkB, tropomyosin-related kinase B; ERK, extracellular signal-regulated kinase; p-, phosphorylated; IP, immunoprecipitation; IB, immunoblotting.

A

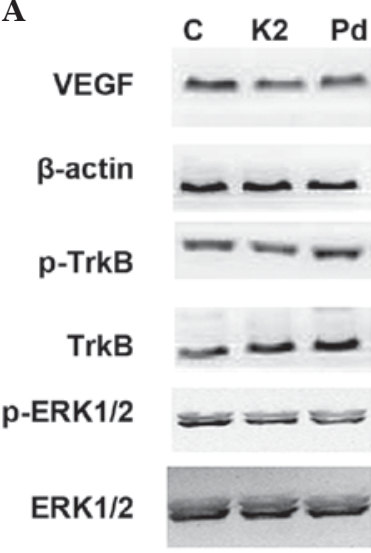

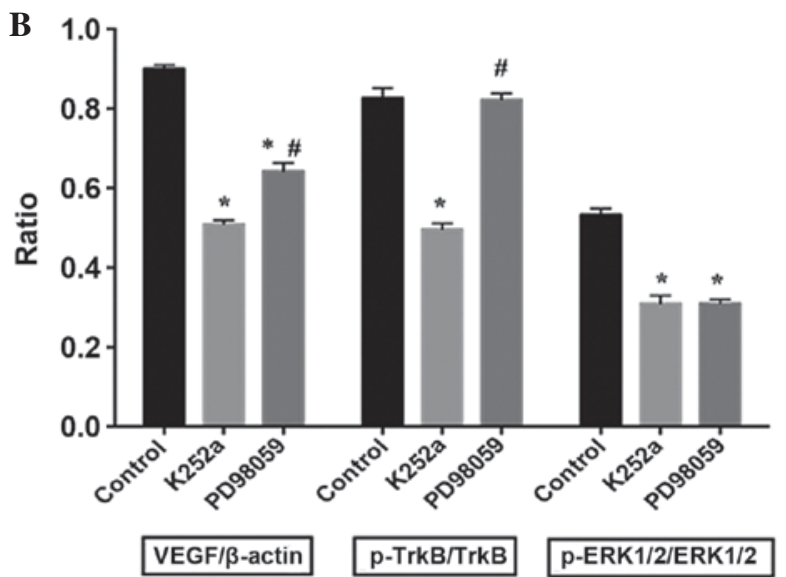

C

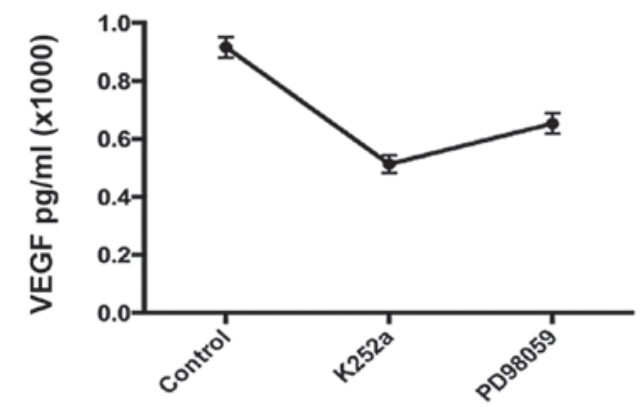

Figure 4. Effects of K252a and PD98059 on the phosphorylation of TrkB and ERK1/2, and expression of VEGF in BDNF-treated osteoblasts. K252a (200 nM) or PD98059 $(25 \mu \mathrm{M})$ were respectively added to the culture medium $30 \mathrm{~min}$ prior to BDNF stimulation. The expression of VEGF was detected $12 \mathrm{~h}$ following BDNF stimulation. (A) Expression of VEGF, and activation of TrkB and ERK1/2 with (B) results of statistical analysis. (C) Concentrations of VEGF in the media were detected using ELISA. Data are presented as the mean \pm standard error of the mean. ${ }^{*} \mathrm{P}<0.05$, compared with the control group; ${ }^{"} \mathrm{P}<0.05$, compared with the K252a-treated group. C, control; K2, K252a; Pd, PD98059; BDNF, brain-derived neurotrophic factor; TrkB, tropomyosin-related kinase B; ERK, extracellular signal-regulated kinase; p-, phosphorylated; VEGF, vascular endothelial growth factor.

time-dependent effect, $50 \mathrm{ng} / \mathrm{ml}$ of BDNF was used to stimulate osteoblasts for 0,3, 6, 12, 24 and 48 h. As shown in Fig. 1A, the expression of VEGF gradually increased and peaked at $12 \mathrm{~h}$. No significant differences were found in expression levels at 12, 24 or $48 \mathrm{~h}$ (Fig. 1B). Similar to protein expression, the ELISA results revealed comparable concentrations 
of VEGF released in the medium (Fig. 1C). To investigate the dose-dependent effect, the cells were stimulated for $12 \mathrm{~h}$ with BDNF at concentrations of 0,25, 50, 100 and $200 \mathrm{ng} / \mathrm{ml}$, respectively. As shown in Fig. 2A-C, maximal expression and secretion of VEGF were observed at a BDNF concentration of $50 \mathrm{ng} / \mathrm{ml}$. Therefore, for subsequent experiments, a BDNF concentration of $100 \mathrm{ng} / \mathrm{ml}$ was used for a duration of $12 \mathrm{~h}$.

Detection of TrkB in osteoblasts. Immunoblotting and immunofluoresence were performed to detect the expression and distribution of TrkB in osteoblasts. The results revealed that TrkB was expressed at a high level in the cells, predominantly located in the cytoplasm and cytomembrane (data not shown).

In BDNF-stimulated osteoblasts, the activities of TrkB and ERK1/2 are increased. To confirm the association between protein kinases in signaling pathways involved in the BDNF-stimulated release of VEGF, the present study detected the phosphorylation levels of ERK1/2 and TrkB separately. BDNF $(50 \mathrm{ng} / \mathrm{ml})$ was used to stimulate the osteoblasts for $0,0.5,1,2,5$ and $10 \mathrm{~min}$, respectively. The results of the statistical analysis revealed that ERK1/2 (Fig 3A and B) and TrkB (Fig. 3C and D) were rapidly activated following BDNF stimulation, and peaked at $1 \mathrm{~min}$.

TrkB is involved in the regulation of VEGF through the activation of ERK1/2 in BDNF-stimulated osteoblasts. In the present study, K252a, a specific inhibitor of TrkB, was used to pretreat the osteoblasts. The results showed that K252a significantly inhibited the phosphorylation of ERK1/2 (Fig. 4A and B) and inhibited the BDNF-induced expression and release of VEGF, (Fig. 4C). To further confirm the role of ERK1/2, PD98059, the ERK1/2 specific inhibitor, was used for pretreatment of the cells. The results demonstrated that PD98059 had the same effect on VEGF as K252a, however, no significant changes were observed in the activity of TrkB (Fig. 4).

\section{Discussion}

The present study investigated the expression and distribution of TrkB in rat calvarial osteoblasts in vitro, and confirmed that BDNF regulated the expression and release of VEGF through activation of the TrkB/ERK1/2 pathway.

Fracture healing is a complex process, which requires the involvement of several growth factors in a temporospatial manner. Using a mouse fracture healing model, Asaumi et al (15) first demonstrated that BDNF was expressed at high levels and localized in osteoblast-like cells, and another study found that the mRNA expression and protein distribution of BDNF and its specific receptor TrkB were detected in osteoblast-like cells obtained from fracture gaps in a human healing diaphyseal fracture (16). However, the mechanism of BDNF in regulating fracture healing remains to be elucidated. The present study demonstrated that BDNF promoted the expression and secretion of VEGF from osteoblasts in a time- and dose-dependent manner. VEGF is one of the most extensively investigated angiogenic growth factors during fracture repair. Inhibition of the activity of VEGF by a VEGF antagonist results in impaired healing of femoral fractures and cortical bone loss in mice (20). In BDNF signaling, several studies have shown that VEGF is involved through the regulation of angiogenesis only. For example, Lin et al (9) found that BDNF can promote the expression of VEGF in human chondrosarcoma cells, and that knockdown of BDNF significantly reduces the expression of VEGF and angiogenesis in vivo. In multiple myeloma, the stable interference of BDNF in multiple myeloma cells significantly inhibits osteolytic bone destruction, and reduces angiogenesis and tumor burden (17). However, compared with multiple myeloma and chondrosarcoma cells, the effects of BDNF on the promotion of the expression and release of VEGF from osteoblasts are more evident. Thus, it can be concluded that BDNF has a positive role in fracture healing by controlling VEGF-mediated angiogenesis.

For transmitting signals, BDNF interacts with its receptors, including high affinity TrkB, low affinity p75 neurotrophin receptor (NTR) and integrin $\alpha 9 \beta 1(7,21)$. In the present study, the expression, distribution and function of TrkB were investigated. Consistent with prior immunohistological staining (data not shown), TrkB was expressed at a high level in osteoblasts, and was located predominantly in the cytoplasm and cytomembrane, and these characteristics made it possible to transmit the effects of BDNF $(15,16)$. In addition to TrkB signaling, the p75 NTR and integrin $\alpha 9 \beta 1$ pathways are also involved in VEGF-mediated angiogenesis. A previous study found that p75 $\left(\mathrm{NTR}^{-/}\right)$mice had reduced hypoxia-inducible factor-1 $\alpha$ stabilization, expression of VEGF and angiogenesis following retinal hypoxia (22), whereas Walsh et al (23) demonstrated that integrin $\alpha 9 \beta 1$ promotes pathological angiogenesis in glioma through direct interaction with neurotrophic factor. Therefore, further investigations are required to elucidate the roles of p75 NTR and integrin $\alpha 9 \beta 1$, and the interaction among the three receptors, in VEGF-mediated angiogenesis during fracture healing.

Following binding of BDNF, three downstream cascades of TrkB can be activated, including PLC $\gamma$, AKT and ERK1/2 (7). PLC $\gamma$, a member of the PLC serine/threonine family, has been shown to mediate the expression of VEGF and angiogenesis in human chondrosarcoma cells following exposure to BDNF/TrkB, whereas in BDNF-treated ECs, the activation of Akt is a key step for angiogenic tube formation $(9,11)$. In the present study, it was demonstrated that the ERK1/2 inhibitor antagonized the BDNF-mediated expression and release of VEGF, suggesting that the activation of ERK1/2 is essential in the BDNF-induced expression and secretion of VEGF in osteoblasts. In addition, the results of the present and previous studies indicated that the above three proteins of the BDNF/TrkB cascade may produce a feedback effect in TrkB signaling $(7,24)$, although PD98059 had no effect on the level of TrkB phosphorylation in the present study. Taken together, the results of the present study provided evidence that BDNF upregulated the expression and secretion of VEGF in rat calvarial osteoblasts via the TrkB receptor and ERK1/2 signaling pathways.

In conclusion, the present study demonstrated for the first time, to the best of our knowledge, that during fracture healing, BDNF promoted the expression and secretion of VEGF in osteoblasts through TrkB and subsequent ERK1/2 activation.

\section{Acknowledgements}

This study was supported by the National Natural Science Foundation of China (grant nos. 81401795 and 81401793), 
the Fundamental Research Funds for the Central Universities (grant no. 20620140712) and the Key Project supported by the Medical Science and Technology Development Foundation, Nanjing Department of Health (grant no. YKK14076).

\section{References}

1. Beamer B, Hettrich C and Lane J: Vascular endothelial growth factor: An essential component of angiogenesis and fracture healing. HSS J 6: 85-94, 2010.

2. Zhu K, Jiao H, Li S, Cao H, Galson DL, Zhao Z, Zhao X, Lai Y, Fan J, Im HJ, et al: ATF4 promotes bone angiogenesis by increasing VEGF expression and release in the bone environment. J Bone Miner Res 28: 1870-1884, 2013.

3. Rui Z, Li X, Fan J, Ren Y, Yuan Y, Hua Z, Zhang N and Yin G: GIT1Y321 phosphorylation is required for ERK1/2and PDGF-dependent VEGF secretion from osteoblasts to promote angiogenesis and bone healing. Int J Mol Med 30: 819-825, 2012.

4. Steinbrech DS, Mehrara BJ, Saadeh PB, Chin G, Dudziak ME, Gerrets RP, Gittes GK and Longaker MT: Hypoxia regulates VEGF expression and cellular proliferation by osteoblasts in vitro. Plast Reconstr Surg 104: 738-747, 1999.

5. Kim IS, Song JK, Zhang YL, Lee TH, Cho TH, Song YM, Kim DK, Kim SJ, Hwang SJ: Biphasic electric current stimulates proliferation and induces VEGF production in osteoblasts. Biochim Biophys Acta 1763: 907-916, 2006.

6. Chen CY, Su CM, Hsu CJ, Huang CC, Wang SW, Liu SC, Chen WC, Fuh LJ and Tang $\mathrm{CH}$ : CCN1 promotes VEGF production in osteoblasts and induces endothelial progenitor cells angiogenesis by inhibiting miR-126 expression in rheumatoid arthritis. J Bone Miner Res 32: 34-45, 2017.

7. Zhang Z, Fan J, Ren Y, Zhou W and Yin G: The release of glutamate from cortical neurons regulated by BDNF via the TrkB/Src/PLC- $\gamma 1$ pathway. J Cell Biochem 114: 144-151, 2013.

8. Zhang Z, Zhou W, Fan J, Ren Y and Yin G: G-protein-coupled receptor kinase interactor-1 serine 419 accelerates premature synapse formation in cortical neurons by interacting with $\mathrm{Ca}(2+) /$ calmodulin-dependent protein kinase II $\beta$. Brain Res Bull 95: 70-77, 2013.

9. Lin CY, Hung SY, Chen HT, Tsou HK, Fong YC, Wang SW and Tang CH: Brain-derived neurotrophic factor increases vascular endothelial growth factor expression and enhances angiogenesis in human chondrosarcoma cells. Biochem Pharmacol 91: 522-533, 2014

10. Binch AL, Cole AA, Breakwell LM, Michael AL, Chiverton N, Cross AK and Le Maitre CL: Expression and regulation of neurotrophic and angiogenic factors during human intervertebral disc degeneration. Arthritis Res Ther 16: 416, 2014.
11. Usui T, Naruo A, Okada M, Hayabe $\mathrm{Y}$ and Yamawaki $\mathrm{H}$ : Brain-derived neurotrophic factor promotes angiogenic tube formation through generation of oxidative stress in human vascular endothelial cells. Acta Physiol (Oxf) 211: 385-394, 2014.

12. Feng N, Huke S, Zhu G, Tocchetti CG, Shi S, Aiba T, Kaludercic N, Hoover DB, Beck SE, Mankowski JL, et al: Constitutive $\mathrm{BDNF} /$ TrkB signaling is required for normal cardiac contraction and relaxation. Proc Natl Acad Sci USA 112: 1880-1885, 2015.

13. Pinski J, Weeraratna A, Uzgare AR, Arnold JT, Denmeade SR and Isaacs JT: Trk receptor inhibition induces apoptosis of proliferating but not quiescent human osteoblasts. Cancer Res 62: 986-989, 2002.

14. Deng FY, Tan LJ, Shen H, Liu YJ, Liu YZ, Li J,Zhu XZ, Chen XD, Tian Q, Zhao M and Deng HW: SNP rs6265 regulates protein phosphorylation and osteoblast differentiation and influences BMD in humans. J Bone Miner Res 28: 2498-2507, 2013.

15. Asaumi K, Nakanishi T, Asahara H, Inoue $H$ and Takigawa $M$ : Expression of neurotrophins and their receptors (TRK) during fracture healing. Bone 26: 625-633, 2000.

16. Kilian O,Hartmann S, Dongowski N, Karnati S, Baumgart-VogtE, Härtel FV, Noll T, Schnettler R and Lips KS: BDNF and its TrkB receptor in human fracture healing. Ann Anat 196: 286-295, 2014.

17. Ai LS, Sun CY, Wang YD, Zhang L, Chu ZB, Qin Y, Gao F, Yan H, Guo T, Chen L, et al: Gene silencing of the BDNF/TrkB axis in multiple myeloma blocks bone destruction and tumor burden in vitro and in vivo. Int J Cancer 133: 1074-1084, 2013.

18. Lam CT, Yang ZF, Lau CK, Tam KH, Fan ST and Poon RT: Brain-derived neurotrophic factor promotes tumorigenesis via induction of neovascularization: Implication in hepatocellular carcinoma. Clin Cancer Res 17: 3123-3133, 2011.

19. Hammond JB and Kruger NJ: The bradford method for protein quantitation. Methods Mol Biol 3: 25-32, 1988.

20. Street J, Bao M, deGuzman L, Bunting S, Peale FV Jr, Ferrara N, Steinmetz H, Hoeffel J, Cleland JL, Daugherty A, et al: Vascular endothelial growth factor stimulates bone repair by promoting angiogenesis and bone turnover. Proc Natl Acad Sci USA 99: 9656-9661, 2002.

21. Staniszewska I, Sariyer IK, Lecht S, Brown MC, Walsh EM, Tuszynski GP, Safak M, Lazarovici P and Marcinkiewicz C: Integrin alpha9 beta1 is a receptor for nerve growth factor and other neurotrophins. J Cell Sci 121: 504-513, 2008.

22. Le Moan N, Houslay DM, Christian F, Houslay MD and Akassoglou K: Oxygen-dependent cleavage of the p75 neurotrophin receptor triggers stabilization of HIF-1 $\alpha$. Mol Cell 44: 476-490, 2011.

23. Walsh EM, Kim R, Del Valle L, Weaver M, Sheffield J, Lazarovici $\mathrm{P}$ and Marcinkiewicz C: Importance of interaction between nerve growth factor and $\alpha 9 \beta 1$ integrin in glial tumor angiogenesis. Neuro Oncol 14: 890-901, 2012.

24. Kommaddi RP, Thomas R, Ceni C, Daigneault K and Barker PA: Trk-dependent ADAM17 activation facilitates neurotrophin survival signaling. FASEB J 25: 2061-2070, 2011. 